\title{
Notes on Calabi-Yau ordinary differential equations
}

\author{
JENG-DAW YU
}

\begin{abstract}
We investigate the structures of Calabi-Yau differential equations and the relations to the arithmetic of the pencils of Calabi-Yau varieties behind the equations. This provides explanations of some observations and computations in the recent paper [6].
\end{abstract}

\section{Introduction}

This note may be regarded as a supplement to the paper [6] of Samol and van Straten. In that paper, the authors study the variation, along a projective line, of the Frobenius action on the cohomology of a Calabi-Yau variety (over a finite field) via the expansion at a totally degenerate point of a certain period of (a lifting of) the pencil. For certain families, they provide a $p$-adic analytic formula for the unit root as well as the Frobenius polynomial (in the case of rank 4) of each fiber provided the fiber is ordinary. Their explicit computations for those examples also reveal the existence of Dwork-type congruences among the coefficients of the period of such a family.

In this note, we provide some theoretical explanations of the observations and computations in their paper. In particular, we show that certain cases of the Dwork congruences, including the mod $p$ ones, follow from the relative geometry of the family.

This article is organized as follows. In $\S 1$, we explore the notion of ordinary differential equations of Calabi-Yau type over a field of characteristic 0 and derive some basic algebraic properties of them. This part provides a further investigation of abstract Calabi-Yau differential equations. In $\S 2$, families of Calabi-Yau varieties over a projective line with totally degenerate fibers at the origin are introduced. The associated Picard-Fuchs equations of such families provide the geometric source of the Calabi-Yau differential equations. $\S 3$ is devoted to the study of the $\bmod p$ and $p$-adic properties of the Calabi-Yau equations. In particular, we study the relation between the solutions of a Calabi-Yau differential equation and the $p$-adic Hasse invariant of the underlying family. This relation provides an explanation of parts of the Dwork congruences. Unfortunately at this stage, we do not know how to 
deal with these Dwork congruences in general. Finally, a further inspection of the Hadamard products appeared in [6] is given in the Appendix.

\section{Ordinary differential equations of Calabi-Yau type}

This section consists of exercises in the theory of differential modules. For basic references, see [8, Chapter 2]. We recall the notion of differential equations of Calabi-Yau type and derive some basic properties of them. Such equations arise in the study of the Picard-Fuchs equations of pencils of Calabi-Yau varieties with certain degenerations. We postpone the geometric picture to the following sections. Notice that some properties we derived here (e.g., Lemma 1.3) can be obtained more easily if the differential equations are from geometry.

Let $K$ be a field of characteristic 0 with a fixed embedding into $\mathbb{C}$. Denote by $\bar{K} \subset \mathbb{C}$ the algebraic closure of $K$. Let $t$ denote a variable. It will also be regarded as a fixed parameter of the projective line $\mathbb{P}^{1}$ over $K$. Let $\theta=t \frac{\mathrm{d}}{\mathrm{d} t}$ be the usual logarithmic derivation with respect to $t$. In this paper, we will use the highly non-standard convention:

$$
g^{\prime}:=\theta g
$$

for a differentiable function $g=g(t)$ of $t$ throughout the discussion. Now consider an ordinary linear differential operator of order $n \geq 1$ of the form

$$
\mathcal{L}=\theta^{n}+\sum_{i=0}^{n-1} a_{i} \theta^{i}
$$

with coefficients $a_{i}=a_{i}(t) \in K(t)$.

\subsection{The condition (N) and the $\beta$-factor}

Suppose $\mathcal{L}$ is a differential operator of order $n$ of the form (1.1). Consider the following condition on $\mathcal{L}$ :

(N) Null exponents: $\mathcal{L}$ has a regular singularity at the origin $t=0$ (i.e., $\left.a_{i} \in K(t) \cap K \llbracket t \rrbracket\right)$ and the associated indicial polynomial of $\mathcal{L}$ at this point reduces to

$$
s^{n}+\sum_{i=0}^{n-1} a_{i}(0) s^{i}=s^{n} .
$$


For any $\mathcal{L}$ as in (1.1), let formally

$$
\beta:=\exp \left(\frac{2}{n} \int a_{n-1} \frac{\mathrm{d} t}{t}\right)
$$

(i.e., a non-zero solution of $n \beta^{\prime}=2 a_{n-1} \beta$ ). We call it the $\beta$-factor of $\mathcal{L}$. It exists at least in some differential field extension of $\bar{K}(t)$.

Lemma 1.1. Suppose $\mathcal{L}$ as in (1.1) satisfies the condition (N). Then we have the following.

(i) The $\beta$-factor in (1.3) can be taken from $(1+t K \llbracket t \rrbracket)$.

(ii) Regard $\mathcal{L}$ as a differential operator in $\bar{K}(t)[\theta]$. Then the differential Galois group of $\mathcal{L}$ is contained in $\operatorname{SL}(n)_{\bar{K}}$ if and only if $\beta$ can be chosen with

$$
\sqrt{\beta^{n}} \in K(t) \cap(1+t K \llbracket t \rrbracket)
$$

Proof. By (1.2), $a_{n-1}(0)=0$ and hence one can formally choose

$$
\int a_{n-1} \frac{\mathrm{d} t}{t} \in t K \llbracket t \rrbracket
$$

Thus the first assertion follows. The differential Galois group of $\mathcal{L} \in \bar{K}(t)[\theta]$ is in $\mathrm{SL}(n)$ if and only if there is a non-zero solution in $\bar{K}(t)$ of the operator $\theta+a_{n-1}([8$, Exercise 1.35.5]). Thus one can require that

$$
\beta \in \sqrt[2 / n]{\bar{K}(t)} \cap(1+t K \llbracket t \rrbracket)
$$

and (ii) follows accordingly.

From now on, we will always assume that $\beta \in(1+t K \llbracket t \rrbracket)$ if $\mathcal{L}$ satisfies the condition $(\mathrm{N})$.

\subsection{The self-adjointness and the polarization}

Let $\mathcal{L}$ be as in (1.1). Recall that the formal adjoint $\mathcal{L}^{*}$ of $\mathcal{L}$ is the differential operator

$$
\mathcal{L}^{*}=(-1)^{n} \theta^{n}+\sum_{i=0}^{n-1}(-1)^{i} \theta^{i} a_{i}
$$


We say that $\mathcal{L}$ is self-adjoint if, as elements in $K(t)[\theta]$,

$$
\mathcal{L}^{*}=(-1)^{n} \beta \mathcal{L} \beta^{-1},
$$

where $\beta$ is defined in (1.3).

For any $\mathcal{L}$ of order $n$, denote by $\mathcal{M}_{\mathcal{L}}$ the left $K(t)[\theta]$-module with a generator $\eta$ defined by

$$
\begin{aligned}
K(t) & \rightarrow \mathcal{M}_{\mathcal{L}}:=K(t)[\theta] / K(t)[\theta] \mathcal{L} \\
1 & \mapsto \eta,
\end{aligned}
$$

where the map is the natural projection. As a $K(t)$-module, $\mathcal{M}_{\mathcal{L}}$ is free of rank $n$ with a basis $\left\{\eta^{(i)}\right\}_{i=0}^{n-1}$, where $\eta^{(i)}:=\theta^{i} \eta$. An element $x \in \mathcal{M}_{\mathcal{L}}$ is called horizontal if $x^{\prime}:=\theta x=0$.

Define a filtration Fil ${ }^{\bullet}$ on $\mathcal{M}_{\mathcal{L}}$ by setting $\mathrm{Fil}^{i}=$ the $K(t)$-submodule generated by $\left\{\eta^{(j)}\right\}_{j=0}^{n-1-i}$. A polarization on $\mathcal{M}_{\mathcal{L}}$ is a $K(t)$-linear, $(-1)^{n+1}$ symmetric, non-degenerate horizontal pairing

$$
\langle,\rangle: \mathcal{M}_{\mathcal{L}} \times \mathcal{M}_{\mathcal{L}} \rightarrow K(t)
$$

such that $\left\langle\mathrm{Fil}^{i}, \mathrm{Fil}^{n-i}\right\rangle=0$ for $0 \leq i \leq(n-1)$. As usual, we say $\mathcal{M}_{\mathcal{L}}$ is polarizable if there exists a polarization on it; $\mathcal{M}_{\mathcal{L}}$ is called polarized if it is equipped with an underlying polarization. The aim of this subsection is to prove the following.

Theorem 1.2. Let $\mathcal{L}$ be as in (1.1) and $\beta$ be defined in (1.3). Then $\mathcal{L}$ is self-adjoint with $\beta \in K(t)$ if and only if $\mathcal{M}_{\mathcal{L}}$ is polarizable.

We first prove the following.

Lemma 1.3. Let $\langle$,$\rangle be a K(t)$-linear horizontal pairing on $\mathcal{M}_{\mathcal{L}}$ such that $\left\langle\eta, \eta^{(i)}\right\rangle=0$ for $0 \leq i<(n-1)$. Let $\gamma=\left\langle\eta, \eta^{(n-1)}\right\rangle$. We have

(i) The pairing is uniquely determined by $\gamma$ and $\gamma=c \beta^{-1}$ for some $c \in K$.

(ii) The pairing is $(-1)^{n+1}$-symmetric.

(iii) The pairing is a polarization if $\gamma \neq 0$.

Proof. Since $\langle$,$\rangle is horizontal, the values \left\langle\eta, \eta^{(i)}\right\rangle, 0 \leq i \leq m$ uniquely determine $\left\langle\eta^{(i)}, \eta^{(j)}\right\rangle$ for all $i+j \leq m$ by a simple inductive procedure of taking derivatives. Thus in our case, the pairing is uniquely determined by $\gamma$. We have $\left\langle\mathrm{Fil}^{i}, \mathrm{Fil}^{n-i}\right\rangle=0$ for $0 \leq i \leq(n-1)$ and the pairing is trivial if $\gamma=0$. 
Since $\left\langle\eta, \eta^{(n-2)}\right\rangle=0$, we have

$$
\begin{aligned}
0 & =\left\langle\eta, \eta^{(n-2)}\right\rangle^{\prime} \\
& =\left\langle\eta^{\prime}, \eta^{(n-2)}\right\rangle+\left\langle\eta, \eta^{(n-1)}\right\rangle,
\end{aligned}
$$

which implies that $\left\langle\eta^{\prime}, \eta^{(n-2)}\right\rangle=-\gamma$. Similarly one finds, for $0 \leq i \leq n-1$, that

$$
\left\langle\eta^{(i)}, \eta^{(n-1-i)}\right\rangle=(-1)^{i} \gamma
$$

by induction. In particular, the pairing is non-degenerate if $\gamma \neq 0$.

Assume $n=2 l$ is even. Then

$$
\begin{aligned}
\gamma & =\left\langle\eta, \eta^{(n-1)}\right\rangle \\
= & -\left\langle\eta^{\prime}, \eta^{(n-2)}\right\rangle \\
& \vdots \\
= & (-1)^{l-1}\left\langle\eta^{(l-1)}, \eta^{(l)}\right\rangle .
\end{aligned}
$$

Taking derivatives of the $l$ equations above, rewriting $\eta^{(n)}$ in terms of $\left\{\eta^{(i)}\right\}_{i=0}^{n-1}$ via $\mathcal{L}$ and summing them up, one gets

$$
l \gamma^{\prime}=-a_{n-1} \gamma
$$

Thus by definition, $\gamma=c \beta^{-1}$ for some $c \in K$. The computation is similar for $n$ odd.

We now prove the parity of $\langle$,$\rangle . We may assume that the pairing is non-$ degenerate. Let $\mathcal{N}=\operatorname{Hom}_{K(t)}\left(\mathcal{M}_{\mathcal{L}}, K(t)\right)$ and consider the natural pairing

$$
(,): \mathcal{N} \times \mathcal{M}_{\mathcal{L}} \rightarrow K(t)
$$

given by $(f, m)=f(m)$. We equip $\mathcal{N}$ with the differential module structure such that $($, ) is horizontal. Then indeed, as a $K(t)[\theta]$-module, $\mathcal{N}$ is generated by $\xi$ with

$$
\left(\xi, \eta^{(i)}\right)= \begin{cases}1 & \text { if } i=n-1 \\ 0 & \text { otherwise }\end{cases}
$$

$\left(\left[8\right.\right.$, Exercise 2.12.6]). Denote $\xi^{(i)}=\theta^{i} \xi$. By a similar computation as above, one deduces that

$$
\left(\xi^{(i)}, \eta^{(j)}\right)= \begin{cases}(-1)^{i} & \text { if } i+j=n-1 \\ 0 & \text { otherwise }\end{cases}
$$


Now the non-degenerate paring $\langle$,$\rangle induces an isomorphism f$ between $\mathcal{M}_{\mathcal{L}}$ and $\mathcal{N}$ and it sends $\eta$ to $\gamma \xi$. Thus under $f$, we can regard $\left\{\xi^{(i)}\right\}_{i=0}^{n-1}$ as another basis of $\mathcal{M}_{\mathcal{L}}$. Moreover, since $\mathcal{N}$ and $\mathcal{M}_{\mathcal{L}}$ are dual to each other (indeed, $\mathcal{N}=\mathcal{M}_{\mathcal{L}^{*}}$ ), after switching the roles of $\mathcal{M}_{\mathcal{L}}$ and $\mathcal{N}$, we see that

$$
\left\langle\xi^{(i)}, \eta^{(j)}\right\rangle=(-1)^{n-1}\left\langle\eta^{(j)}, \xi^{(i)}\right\rangle .
$$

This completes the proof of the parity of $\langle$,$\rangle .$

Proof of Theorem 1.2. Suppose $\mathcal{L}$ is self-adjoint and $\beta \in K(t)$. Then by the lemma above, we can equip $\mathcal{M}_{\mathcal{L}}$ with the polarization determined by setting $\left\langle\eta, \eta^{(n-1)}\right\rangle=\beta^{-1}$.

On the other hand, suppose $\mathcal{M}_{\mathcal{L}}$ is polarized. Then $\beta \in K(t)$ by the lemma above. Multiplying by a non-zero constant, we may assume that $\left\langle\eta, \eta^{(n-1)}\right\rangle=\beta^{-1}$. Then $\beta \eta$ is dual to $\eta^{(n-1)}$ with respect to the basis $\left\{\eta^{(i)}\right\}_{i=0}^{n-1}$. Thus $\mathcal{L}^{*} \beta \eta=0\left(\left[8\right.\right.$, Exercise 2.12.6]) and hence $\mathcal{L}^{*}=(-1)^{n} \beta \mathcal{L} \beta^{-1}$.

\subsection{Calabi-Yau differential equations and the $q$-coordinate}

Definition. A differential operator $\mathcal{L} \in K(t)[\theta]$ of form (1.1) is called Calabi-Yau if $\mathcal{L}$ is self-adjoint and satisfies condition (N) in $\S 1.1$.

Remark. It might be better to call such an $\mathcal{L}$ as above locally or quasiCalabi-Yau since in literature (e.g., in $[1,6]$ ), there are some integral conditions on solutions of $\mathcal{L}$ (cf. Theorem 3.3) and here we do not require that $\mathcal{L}$ is regular singular away from 0 . However, there seems to be no unified definition yet.

For any $\mathcal{L}$ as in (1.1) satisfying the condition $(\mathrm{N})$, we set

$$
\Lambda_{\mathcal{L}}=K \llbracket t \rrbracket[\theta] / K \llbracket t \rrbracket[\theta] \mathcal{L} .
$$

It is a $K \llbracket t \rrbracket$-lattice in the completion $\mathcal{M}_{\mathcal{L}} \otimes_{K[t]} K \llbracket t \rrbracket$ of $\mathcal{M}_{\mathcal{L}}$. We abuse the notation by denoting $\mathrm{Fil}^{i} \subset \Lambda_{\mathcal{L}}$ the induced filtration from $\mathrm{Fil}^{i} \subset \mathcal{M}_{\mathcal{L}}$.

Suppose $\mathcal{L}$ is Calabi-Yau. Denote by $F(t)=F_{0}(t)$ the unique formal power series such that

$$
\mathcal{L} F(t)=0, \quad F(t) \in 1+t K \llbracket t \rrbracket
$$


for $n \geq 2$, denote by $F_{i}(t), 1 \leq i \leq n-1$, the solutions with logarithmic pole of the form

$$
\mathcal{L} F_{i}(t)=0, \quad \sum_{r=0}^{i}(-1)^{r} F_{i-r}(t) \cdot \frac{\log ^{r} t}{r !} \in t K \llbracket t \rrbracket .
$$

Thus $\left\{F_{i}\right\}$ forms the Frobenius basis of solutions near $t=0$ of $\mathcal{L}$. Let

$$
w r_{i}=w r\left(F, F_{1}, \ldots, F_{i}\right):=\operatorname{det}\left(F_{r}^{(s)}\right)_{0 \leq r, s \leq i}
$$

be the wronskians of $\left\{F_{r}\right\}_{r=0}^{i}$ and set

$$
q=\exp \left(\frac{F_{1}}{F}\right) \in t+t^{2} K \llbracket t \rrbracket .
$$

Thus $K \llbracket t \rrbracket=K \llbracket q \rrbracket$. We call $q$ the $q$-coordinate of $\mathcal{L}$.

Theorem 1.4. Suppose $\mathcal{L}$ in (1.1) is Calabi-Yau. With the notations above, there exists a unique increasing filtration $U_{\bullet}$ of $K \llbracket t \rrbracket[\theta]$-submodules of $\Lambda_{\mathcal{L}}$ such that, for all $i$,

$$
\Lambda_{\mathcal{L}}=U_{i} \oplus \mathrm{Fil}^{i+1}
$$

and $U_{i+1} / U_{i}$ are trivial $K \llbracket t \rrbracket[\theta]$-modules. Moreover, up to a multiplicative constant, there exists a unique sequence $\left\{u_{i} \in \Lambda_{\mathcal{L}}\right\}_{i=0}^{n-1}$ with the following two properties:

(i) As a $K \llbracket t \rrbracket$-module, $U_{i}$ is generated by $\left\{u_{r}\right\}_{r=0}^{i}$.

(ii) $u_{i}$ is of the form $u_{i}=\sum_{r=0}^{n-1-i} v_{i, r} \eta^{(r)}$ with

$$
v_{i, n-1-i}=\beta \frac{w r_{i}}{w r_{i-1}}
$$

Consequently, we have $u_{i+1}^{\prime}=\tau_{i+1} \cdot u_{i}$, where

$$
\tau_{i+1}=\frac{w r_{i-1} w r_{i+1}}{w r_{i}^{2}}
$$

and in particular, $\tau_{1}=(\log q)^{\prime}$.

Proof. We find $\left\{u_{i}\right\}$ satisfying (1.11) and (1.12) by induction. 
The condition $(\mathrm{N})$ of $\mathcal{L}$ implies that, up to a constant multiple, there is a unique non-zero element

$$
u_{0}=\sum_{r=0}^{n-1} v_{0, r} \eta^{(r)}, v_{0, r} \in K \llbracket t \rrbracket
$$

which is horizontal. Since

$$
\mathcal{L}\left\langle u_{0}, \eta\right\rangle=\left\langle u_{0}, \mathcal{L} \eta\right\rangle=0
$$

we see that $v_{0, n-1}=c \beta F$ for some constant $c$ by Lemma 1.3. It is obvious that $c \neq 0$. Thus after modifying by a constant, $v_{0, n-1}$ is of the form (1.12).

Let $\mathcal{L}^{[i]}$ be the $i$ th exterior product of $\mathcal{L}$. Then $\mathcal{L}^{[i]}$ satisfies the condition $(\mathrm{N})$ and $\Lambda_{\mathcal{L}^{[i]}}$ is a quotient of the $i$ th exterior power of the $K \llbracket t \rrbracket$-module $\Lambda_{\mathcal{L}}$. There exists a unique (up to a scalar) horizontal $u^{[i]} \in \Lambda_{\mathcal{L}^{[i]}}$ of the form

$$
u^{[i]}=\sum_{r_{1} \leq \cdots \leq r_{i}} v_{r_{1} \cdots r_{i}}^{[i]} \eta^{\left(r_{1}\right)} \wedge \cdots \wedge \eta^{\left(r_{i}\right)}
$$

with $v_{n-i, \cdots, n-1}^{[i]} \neq 0$. (Notice that $\eta^{(n-i)} \wedge \cdots \wedge \eta^{(n-1)} \neq 0$ in $\Lambda_{\mathcal{L}^{[i]}}$.) With the induced pairing,

$$
\mathcal{L}^{[i]}\left\langle u^{[i]}, \eta \wedge \cdots \wedge \eta^{(i-1)}\right\rangle=\left\langle u^{[i]}, \mathcal{L}^{[i]}\left(\eta \wedge \cdots \wedge \eta^{(i-1)}\right)\right\rangle=0 .
$$

Thus we have, after modifying by a scalar, $v_{n-i, \cdots, n-1}^{[i]}=\beta^{i} w r_{i} \in K \llbracket t \rrbracket^{\times}$. Therefore, by subtracting an element in $U_{i-1}$, we obtain $u_{i}$ satisfying (1.11) and (1.12).

Corollary 1.5. Suppose $\mathcal{L}$ in (1.1) is Calabi-Yau. With notations in Theorem 1.4, we have $\left\langle U_{i-1}, U_{n-1-i}\right\rangle=0$ and $\tau_{i}=\tau_{n-i}$ for all $1 \leq i \leq n$.

Proof. By the last assertion of Thorem 1.4, we see that $K(t) \otimes_{K[t]} U_{i}$ are the only possible $K((t))[\theta]$-submodules of $K(t) \otimes_{K[t]} \Lambda_{\mathcal{L}}$. Since $U_{i}$ is stable under $\theta$, so is its orthogonal complement $U_{i}^{\perp}$. Thus by a rank counting, $U_{i}^{\perp}=U_{n-2-i}$.

Since $u_{i}$ lies in $\mathrm{Fil}^{i}$ and $\left\langle\mathrm{Fil}^{i}, \mathrm{Fil}^{n-i}\right\rangle=0$, we have $\left\langle u_{i}, u_{n-1-j}\right\rangle=0$ if $i \neq j$ and $\left\langle u_{i}, u_{n-1-i}\right\rangle$ are non-zero constants. Consequently,

$$
\begin{aligned}
0 & =\left\langle u_{i}, u_{n-i}\right\rangle^{\prime} \\
& =\tau_{i} \cdot\left\langle u_{i-1}, u_{n-i}\right\rangle+\tau_{n-i} \cdot\left\langle u_{i}, u_{n-1-i}\right\rangle .
\end{aligned}
$$

Since $\tau_{i}(0)=1$ for all $i$ by definition, the second assertion follows. 


\section{Degenerations}

For the definitions and basic properties of logarithmic structures, see [3].

Fix a base field $k$. Consider a flat projective pencil $\pi: X \rightarrow \mathbb{P}^{1}$ whose generic fiber is smooth. We further assume that each singular fiber of $\pi$ is a union of reduced divisors with normal crossings. For such a pencil $\pi$, we equip $X$ and $\mathbb{P}^{1}$ with the natural logarithmic structures associated to the union of the singular fibers (which is a reduced normal crossing divisor on $X$ ) and the critical values (which form a reduced divisor on $\mathbb{P}^{1}$ ), respectively. Then $\pi$ is log-smooth. Denote by $\omega^{i}=\omega_{X / \mathbb{P}^{1}}^{i}$ the sheaf on $X$ of relative differential $i$-forms with log poles with respect to the log structures.

Now suppose that the generic fiber of $\pi$ is an absolutely irreducible Calabi-Yau variety of dimension $m \geq 1$. We will call such a $\pi$ a nice pencil of Calabi-Yau varieties of dimension $m$. Then the sheaf $\pi_{*} \omega^{m}$ is an invertible sheaf on $\mathbb{P}^{1}$. Suppose there exists a locally direct factor $\mathcal{M}$ of $R^{m} \pi_{*} \omega^{\bullet}$ of rank $(m+1)$ which contains $\pi_{*} \omega^{m}$ and is stable under the Gauss-Manin connection $\nabla$. Now suppose $k=\mathbb{C}$. Let $a \in \mathbb{P}^{1}(\mathbb{C})$ be a $\mathbb{C}$-valued point and let $N$ denote (the logarithm of) the local monodromy around $a$. Then $N$ acts on the stalk $\mathcal{M}_{a}$ at $a$ and is nilpotent. Notice that if $N^{m} \neq 0$ on $\mathcal{M}_{a}$, then $\mathcal{M}$ must be the unique irreducible locally direct factor of $R^{m} \pi_{*} \omega^{\bullet}$ which contains $\pi_{*} \omega^{m}$ and is stable under $\nabla$.

We make the following working definition, which is a special variant of being Hodge-Tate in the sense of Deligne $([2, \S 6])$.

Definition. With notations and assumptions as above, we call $\mathcal{M}$ totally degenerate at $a$ if $N^{m} \neq 0$ on $\mathcal{M}_{a}$. We will abuse the notation by saying that the fiber at $a$ of the family $\pi$ is totally degenerate if such an $\mathcal{M}$ exists. We call $\mathcal{M}$ the degenerate factor of $\pi$ in this case.

Lemma 2.1. Let $\pi: X \rightarrow \mathbb{P}^{1}$ over $\mathbb{C}$ be a nice pencil of Calabi-Yau varieties of dimension $m$ as above with a totally degenerate fiber at 0 . Then the Poincaré pairing is non-degenerate around 0 on the degenerate factor $\mathcal{M}$.

Proof. Let $\eta$ be a local basis of sections of $\pi_{*} \omega^{m}$ near 0 . By assumption, we have, for $0 \leq i \leq m$,

$$
\eta^{(i)} \in \mathrm{Fil}^{m-i} \backslash \mathrm{Fil}^{m-i+1}
$$

and they form a local basis of $\mathcal{M}$ at 0 . Here $\eta^{(i)}=(\nabla(\theta))^{i} \eta\left(=N^{i} \eta\right.$ at 0 ). Since $\left(\mathrm{Fil}^{i}\right)^{\perp}=\mathrm{Fil}^{m+1-i}$, the cup-product $\gamma:=\left\langle\eta, \eta^{(m)}\right\rangle$ is an invertible function near 0. By Lemma 1.3, the assertion follows. 
Corollary 2.2. Let $\pi: X \rightarrow \mathbb{P}^{1}$ over $K \subset \mathbb{C}$ be a nice pencil of Calabi-Yau varieties of dimension $m$ with a totally degenerate fiber at 0 . Let $\eta$ be a local basis of sections of $\pi_{*} \omega^{m}$ at 0 and let $\mathcal{L}$ be the Picard-Fuchs operator of $\eta$. Then $\mathcal{L}$ is a Calabi-Yau differential equation with respect to the parameter $t$ of order $(m+1)$.

Proof. The self-adjointness of $\mathcal{L}$ follows from Lemma 2.1. The validity of condition $(\mathrm{N})$ is obvious by the total degeneracy assumption.

\section{Mod $p$ and $p$-adic aspects}

In this section, we fix a prime $p$ and suppose $p>m$. Let $K$ be a finite unramified extension of $\mathbb{Q}_{p}$ with ring of integers $W$ and residue field $k$. Let $\pi: X \rightarrow \mathbb{P}^{1}$ be a nice pencil of Calabi-Yau varieties of dimension $m$ over $K$ with a totally degenerate fiber at 0 . Let $\omega^{i}$ be the sheaf on $X$ of relative differential $i$-forms with log-poles along the logarithmic structure. We fix a basis $\eta$ near 0 of sections of $\pi_{*} \omega^{m} \subset R^{m} \pi_{*} \omega^{\bullet}$ and let $\mathcal{L}$ be the Picard-Fuchs operator of $\eta$ as before. We call that $\pi$ has nice reduction if $\pi$ has a flat model over $W$ such that (i) the reduction $\bar{\pi}: \bar{X} \rightarrow \mathbb{P}^{1}$ over $k$ is also a nice pencil of Calabi-Yau varieties, and (ii) the two $\log$ structures of $\pi$ and $\bar{\pi}$ are induced from a smooth log structure on the flat model.

\subsection{The $p$-adic input}

Lemma 3.1. Suppose the pencil $\pi$ has nice reduction and the degenerate factor $\mathcal{M}_{0}$ at 0 is stable under (a lifting of) the absolute Frobenius. Then the Frobenius action on $\mathcal{M}_{0}$ is ordinary and consequently $\mathcal{M}$ is generically ordinary.

Proof (cf. [9, Theorem 2.2]). Since $\mathcal{M}$ is totally degenerate at 0 , the Hodge structure on $\mathcal{M}_{0}$ is a successive extension of rank 1 Hodge structures of pairwise different weights. Since the Frobenius respects the Hodge and the weight filtrations (cf. [5, Remarques 3.28]), the result follows.

Lemma 3.2. Suppose the pencil $\pi$ over $K$ with a totally degenerate fiber at 0 has nice reduction and the degenerate factor $\mathcal{M}_{0}$ at 0 is stable under Frobenius. Then there exists a non-zero constant $c \in W$ such that $c \beta F \in$ $W \llbracket t \rrbracket$. 
Proof. By Lemma 3.1 and [4, Proposition 3.1.3], there is a non-zero element

$$
u=\sum_{i=0}^{m} v_{i} \eta^{(i)}, \quad v_{i} \in W \llbracket t \rrbracket
$$

which is horizontal. By Theorem 1.4, $v_{m}=c \beta F$ for some constant $c$.

Theorem 3.3. Let $\pi$ be a nice pencil of Calabi-Yau varieties of dimension $m$ over $\mathbb{Q}$. Fix a basis near 0 of sections of $\pi_{*} \omega^{m}$ and let $\mathcal{L}$ be the associated Picard-Fuchs operator. Let $F$ be the formal solution of $\mathcal{L}$ as in (1.7). Then $F(t) \in\left(1+t \mathbb{Z}_{p} \llbracket t \rrbracket\right)$ for all $p$ sufficiently large. In particular, $F(t) \in \mathbb{Z}[1 / N] \llbracket t \rrbracket$ for some integer $N \neq 0$.

Proof (cf. [6, Conjecture 2.1]). By the lemma above and its proof, we see that except for a finite number of primes, $\beta F \in \mathbb{Z}_{p} \llbracket t \rrbracket$ for all the remaining $p$ such that the pencil $\pi$ has nice reduction. By Lemma $1.3, \beta(t)$ is rational over $\mathbb{Q}$ and hence is in $\left(1+t \mathbb{Z}_{p} \llbracket t \rrbracket\right)$ for almost all $p$. Thus the assertion follows.

\subsection{The Hasse invariant and the differential equation}

Assume that $\pi$ has nice reduction $\bar{\pi}$. Let $\bar{\omega}^{i}$ be the sheaf on $\bar{X}$ of relative differential $i$-forms with log-poles. We choose $\eta$ that can be extended to the flat model of $\pi$ and assume the following condition is satisfied:

(R) Non-degeneracy of the reduction of $\eta$ at the origin:

$$
\left\langle\eta, \eta^{(m)}\right\rangle \in W^{\times}+t W \llbracket t \rrbracket
$$

Let $\sigma$ be the lifting to $W \llbracket t \rrbracket$ of the absolute Frobenius defined by sending $t$ to $t^{p}$. For any $x \in W \llbracket t \rrbracket$, denote by $x^{\sigma}$ the image under $\sigma$.

Consider the adjoint morphism

$$
V: \bar{\pi}_{*} \bar{\omega}^{m} \rightarrow \sigma^{*} \bar{\pi}_{*} \bar{\omega}^{m}
$$

of the absolute Frobenius with respect to the cup-product on $R^{m} \pi_{*} \bar{\omega}^{\bullet}$. Notice that $V$ is horizontal since the Frobenius and the cup-product are. Represent $V$ by $\mathfrak{H}$ defined by

$$
V(\eta)=\mathfrak{H} \cdot \sigma^{*} \eta
$$


With our choice of the parameter, $\mathfrak{H}$ is an element in $k(t)$. We call $\mathfrak{H}$ the Hasse invariant of the family $\bar{\pi}$ (with respect to $\eta$ ). Notice that $\mathfrak{H}(0)$ is well defined and non-zero at $t=0$ by Lemma 3.1 .

Proposition 3.4. We have $\mathcal{L} \mathfrak{H}=0$. That is, with respect to the parameter $t$, the Hasse invariant $\mathfrak{H}$ is a rational solution of $\mathcal{L}$ in characteristic $p$.

Proof. By the very definition, $\nabla(\mathcal{L}) \eta=0$ and $\nabla \sigma^{*} \eta=0$. Since $V$ is horizontal, we see that $\mathcal{L} \mathfrak{H}=0$ by applying $\nabla(\mathcal{L})$ to Equation (3.1).

If (R) is satisfied, then by Lemmas 1.3, 3.2 and their proofs, $F \in W \llbracket t \rrbracket$. Let

$$
\mathcal{H}(t)=\frac{F(t)}{F(t)^{\sigma}}
$$

regarded as a formal power series over $W$. Let $\overline{\mathcal{H}}(t)$ be the reduction mod $p$ of $\mathcal{H}(t)$.

Proposition 3.5. Suppose $\pi$ satisfies the condition (R) above. Then we have the following:

(i) $\overline{\mathcal{H}}(t) \in(1+t k \llbracket t \rrbracket) \cap k(t)$.

(ii) The function $\overline{\mathcal{H}}$ satisfies $\mathcal{L} \overline{\mathcal{H}}=0$.

Proof. With the notations in Theorem 1.4, the element $u_{0}=\beta F \eta^{(m)}+\cdots$ is a local generator of the submodule $U_{0}$ of horizontal sections of the degenerate factor $\mathcal{M}$ (cf. the proof of Lemma 3.2). Then $U_{0}$ is a unit-root $F$-(iso)crystal ([4, Proposition 3.1.3]) and hence by $[4,4.1 .9]$, the function $\beta F /(\beta F)^{\sigma}$ is a lifting of an element in $k(t)$. Thus the first assertion follows.

Formally we have

$$
F(t)=\frac{F(t)}{F(t)^{\sigma}} \frac{F(t)^{\sigma}}{F(t)^{\sigma^{2}}} \cdots=\mathcal{H}(t) \mathcal{H}(t)^{\sigma} \cdots .
$$

Applying the differential operator $\mathcal{L}$, we have

$$
\begin{aligned}
0=\mathcal{L} F(t) & =\mathcal{L}\left(\mathcal{H}(t) \mathcal{H}(t)^{\sigma} \cdots\right) \\
& \equiv(\mathcal{L} \mathcal{H}(t))\left(\mathcal{H}(t)^{\sigma} \cdots\right) \\
& \equiv(\mathcal{L} \overline{\mathcal{H}}(t))\left(\overline{\mathcal{H}}(t)^{p} \cdots\right) \quad(\bmod p) .
\end{aligned}
$$

Thus the rational function $\overline{\mathcal{H}}(t)$ is a solution of $\mathcal{L}$ in characteristic $p$. 
Proposition 3.6. Assume condition $(R)$ is fulfilled. Let $c=\mathfrak{H}(0)$. We have $\mathfrak{H}=c \overline{\mathcal{H}}$ regarded as rational functions of $t$.

Proof. Over a non-empty open subset of $\mathbb{P}^{1}$, both functions $\mathfrak{H}$ and $c \overline{\mathcal{H}}$ represent the absolute Frobenius action on $\eta$ ([4, 4.1.9]; cf. the proof of Proposition 3.5(i)). Thus the assertion follows.

Corollary 3.7. Assume that condition $(R)$ is fulfilled and $\pi_{*} \omega^{m}=\mathcal{O}(1)$. Suppose $\eta \in \Gamma\left(\mathbb{P}^{1}, \pi_{*} \bar{\omega}^{m}\right)$ and let $c=\mathfrak{H}(0)$. Then $c \overline{\mathcal{H}}(t)=\mathfrak{H}(t) \equiv c F^{<p}(t)$ $\bmod p$, where $F^{<p}(t)$ is the truncation of $F(t)$ up to degree $(p-1)$.

Proof. Under the assumptions, $\mathfrak{H}(t)$ is a polynomial of degree $<p$.

Remark. The statement of the corollary is equivalent to the $\bmod p$ case of the Dwork congruences of the coefficients $\chi(n)$ of $F(t)=\sum \chi(n) t^{n}$ in $[6$, $\S 2.3]$. Indeed let $\nu=\sum_{i \geq 0} \nu_{i} p^{i}, 0 \leq \nu_{i}<p$, be the $p$-adic expansion of an integer $\nu \geq 0$. Then Corollary 3.7 implies

$$
\chi(\nu) \equiv \prod_{i \geq 0} \chi\left(\nu_{i}\right) \quad \bmod p,
$$

which is equivalent to the $\bmod p$ case.

On the other hand, if $\pi_{*} \omega^{m}=\mathcal{O}(n)$ for some $n>1$, then $\overline{\mathcal{H}}$ is of higher degree. This may be used to detect the degree of $\pi_{*} \omega^{m}$ from the period $F$.

\subsection{The higher congruences}

We keep the assumptions and notations in Lemma 3.1; assume that $\pi$ has nice reduction $\bar{\pi}$ and $(\mathrm{R})$ is fulfilled.

In this subsection, we are interested in understanding the higher Dwork congruences geometrically. For this purpose, we suppose that $\pi_{*} \omega^{m}=\mathcal{O}(1)$ over $W$ and $\eta \in \pi_{*} \omega^{m}\left(\mathbb{P}^{1}\right)$ is globally defined. Thus $\beta^{-1}$, which equals $\left\langle\eta, \eta^{(m)}\right\rangle$ up to a multiplicative constant, is in $W[t]$.

We use the following notations. Let $\mathcal{H}_{0}=F^{<p}(t)$. Denote by $\Delta_{0}=$ Spec $k[t] / \overline{\mathcal{H}}$ the Hasse locus of the pencil $\bar{\pi}$. Let $\Delta_{\infty}=\left(\mathbb{A}_{W}^{1}\right) / \Delta_{0}$ be the completion of the affine line $\mathbb{A}_{W}^{1}$ along $\Delta_{0}$. Define

$$
R_{\infty}={\underset{\lim }{n}}_{n} W\left[t, \mathcal{H}_{0}^{-1}\right] /\left(p^{n+1}\right)
$$

and let

$$
S_{\infty}=\operatorname{Spf} R_{\infty}
$$


Under this circumstance, $\mathcal{H} \in R_{\infty}$ is a section of $\mathcal{O}(p-1)$ with $\mathcal{H} \equiv \mathcal{H}_{0}$ $\bmod p$ (cf. Corollary 3.7 and [4, 4.1.9]; locally $\mathcal{H}$ defined in (3.2) represents the absolute Frobenius on $\beta^{-1} u$ up to a multiplicative constant, where $u$ occurs in the proof of Lemma 3.2). On the other hand, the unit-root part of $\mathcal{M}$ defines a (multiplicative) formal group over $S_{\infty}$.

We make a further assumption:

(E) The above formal group over $S_{\infty}$ can be extended to a formal group $G$ over the completion $\left(\mathbb{P}_{W}^{1}\right) / \mathbb{P}_{k}^{1}$.

For example [9], the Dwork pencil has certain degenerate fibers; however, the attached formal group can be extended to the whole $\left(\mathbb{P}_{W}^{1}\right)_{/ \mathbb{P}_{k}^{1}}$ in an explicit way by writing down its logarithm (see [9, Proposition 5.2]).

Now suppose (E) holds. Represent $G$ by a formal group law over the affine part $\left(\mathbb{A}_{W}^{1}\right) / \mathbb{A}_{k}^{1}$ with logarithm

$$
l(\tau)=\sum_{n=1}^{\infty} \alpha(n) \frac{\tau^{n}}{n}, \quad \alpha(n) \in \varliminf_{\varlimsup} W[t] /\left(p^{i+1}\right),
$$

which is normalized by $\alpha\left(p^{n}\right) \equiv \mathcal{H} \alpha\left(p^{n-1}\right)^{\sigma} \bmod p^{n}$ for all $n \geq 1$. Write $F(t)=\sum \chi(n) t^{n}$. For any $1 \leq m<p$ and $s \geq 0$, consider the truncation of $F(t)$

$$
F_{\left(m p^{s}\right)}=\sum_{n=(m-1) p^{s}}^{m p^{s}-1} \chi(n) t^{n} .
$$

Lemma 3.8. With notations as above, let $\left\{a_{n}\right\}_{n=0}^{\infty}, a_{n} \in W[t]$, be a sequence with $a_{0} \in W^{\times}$and $a_{n+1} \equiv \mathcal{H} a_{n}^{\sigma} \bmod p^{n+1}$ for all $n \geq 0$. Then we have the following:

(i) For any $b \in W[t]$ with $b \equiv a_{1} \bmod p$, there exists a sequence $\left\{b_{n}\right\}_{n=1}^{\infty}$, $b_{n} \in W[t]$, such that $b_{1}=b$ and $b_{n+1} \equiv \mathcal{H} b_{n}^{\sigma} \bmod p^{n+1}$ for all $n \geq 1$.

(ii) Fix a positive integer $n$. For any $c \in W[t]$ with $c \equiv a_{n} \bmod p^{n}$, there exists an element $\tilde{c} \in W[t]$, unique modulo $p^{n+1}$, such that

$$
a_{n+1} \cdot c^{\sigma} \equiv a_{n}^{\sigma} \cdot \tilde{c} \bmod p^{n+1} .
$$

Proof. (i) Indeed, if $b=a_{1}+p \delta$, one checks that the sequence of elements

$$
b_{n}=a_{n}+p \cdot a_{n-1} \delta^{\sigma^{n-1}}, \quad n \geq 2
$$

(with $b_{1}=b$ ) satisfies the requirement. 
(ii) The assumption implies $a_{n+1} \equiv a_{1}^{1+p+\cdots+p^{n}} \bmod p$. Write $c=a_{n}+$ $p^{n} \alpha$. We are asked to solve $\varepsilon$ in

$$
a_{n+1}\left(a_{n}+p^{n} \alpha\right)^{\sigma} \equiv a_{n}^{\sigma}\left(a_{n+1}+p^{n} \varepsilon\right) \quad \bmod p^{n+1},
$$

which is equivalent to solve

$$
a_{n}^{\sigma} \varepsilon \equiv a_{n+1} \alpha^{\sigma} \bmod p
$$

The latter has a unique solution $a_{1} \cdot \alpha^{\sigma}$ modulo $p$.

Theorem 3.9. With notations as above and under the assumption (E), the formal power series

$$
\tilde{l}(\tau)=\sum_{1 \leq m<p}^{s \geq 0} F_{\left(m p^{s}\right)} \frac{\tau^{m p^{s}}}{m p^{s}}
$$

is the logarithm of a formal group law $\tilde{G}$ over $\left(\mathbb{A}_{W}^{1}\right) / \mathbb{A}_{k}^{1}$. Moreover, $\tilde{G}$ is strictly isomorphic to $G$ defined above over $S_{\infty}$.

Proof. We shall show that the sequence $\left\{F_{\left(m p^{s}\right)}\right\}$ satisfies the congruence property

$$
F_{\left(m p^{s}\right)} \equiv \mathcal{H} \cdot F_{\left(m p^{s-1}\right)}^{\sigma} \bmod p^{s}
$$

Consequently, the theorem follows by [7, Theorem (A.8), (A.9)].

For $s=1$, the statement is a consequence of Corollary 3.7.

Away from $\Delta_{\infty}$, we have

$$
\alpha(m, s):=\frac{\alpha\left(m p^{s}\right)}{\alpha\left(m p^{s-1}\right)^{\sigma}} \in R_{\infty}
$$

and $\alpha(m, s) \equiv \mathcal{H} \bmod p^{s}$ for all $s \geq 1$. To shorten the notation, first assume that $\mathcal{H} \bmod p$ is exactly of degree $(p-1)$. Now since $\alpha(m, s)$ represents the absolute Frobenius modulo $p^{s}$, which is a section of the sheaf $\mathcal{O}(p-1)$ of $\mathbb{P}^{1}$ over the affine open Spec $W\left[t, \mathcal{H}_{0}^{-1}\right] /\left(p^{s}\right)$, we have

$$
\alpha(m, s) \equiv \frac{A}{\mathcal{H}_{0}^{n}} \quad \bmod p^{s}
$$

for some $n$ and $A \in W[t] /\left(p^{s}\right)$ of degree $(n+1)(p-1)$. 
On the other hand, applying Lemma 3.8(i) with $a_{n}=\alpha_{1, n}$ and $b=$ $F_{(m p)} / F_{(m)}(\in W[t]$ with invertible constant term), we obtain a sequence $\left\{b_{n}\right\}$ such that, for $s \geq 1$,

$$
\alpha(m, s) \equiv \frac{b_{s}}{b_{s-1}^{\sigma}} \equiv \mathcal{H} \quad \bmod p^{s} .
$$

(Here we set $\left.b_{0}=F_{(m)}.\right)$ By the first congruence in (3.5) together with (3.4), we see that $\operatorname{deg} b_{s}=m p^{s}-1$. Now by a simple induction argument, the second congruence in (3.5) together with Lemma 3.8(ii) (for $\left\{b_{n}\right\}$ ) imply that one can take $b_{s}=F_{\left(m p^{s}\right)}$ for all $s \geq 0$.

In general, we represent the occurred finite degree elements as homogeneous elements in $W\left[t_{0}, t_{1}\right]$ (e.g., $t_{0}^{m p^{s}-1} \alpha\left(t_{1} / t_{0}\right)$ instead of $\alpha$ ) to obtain that $\operatorname{deg} b_{s}<m p^{s}$ and then repeat the argument above. This completes the proof.

Corollary 3.10. Write $F(t)=\sum \chi(n) t^{n}$. For all non-negative integers $\nu, m, s$ with $0 \leq \nu<p$, we have

$$
\chi\left(\nu+m p^{s+1}\right) \equiv \chi(\nu) \chi\left(m p^{s}\right) \quad \bmod p^{s+1} .
$$

Proof. One checks readily that it suffices to establish the congruences for $1 \leq m<p$. Assuming $m$ is in this range. The theorem above (see (3.3)) implies that, for all $s \geq 0$,

$$
\left(\kappa_{m, s}\right) \quad F \cdot F_{\left(m p^{s}\right)}^{\sigma} \equiv F_{\left(m p^{s+1}\right)} \cdot F^{\sigma} \bmod p^{s+1} .
$$

For $m=1$, by comparing the coefficients of $t^{\nu+p^{s+1}}$ in both sides of the equation $\left(\kappa_{1, s}\right)$, we get

$$
\sum_{j=0}^{p^{s}-1} \chi\left(\nu+p^{s+1}-p j\right) \chi(j) \equiv \sum_{j=0}^{p^{s}-1} \chi(\nu+p j) \chi\left(p^{s}-j\right) \quad \bmod p^{s+1} .
$$

By canceling common terms on the two sides of the congruence, the assertion (corresponding to $j=0$ ) follows immediately. For $m>1$, by comparing the coefficients of $t^{\nu+m p^{s+1}}$ in both sides of equations $\left(\kappa_{m, s}\right)$ and $\left(\kappa_{m-1, s}\right)$, one obtains the desired congruences by induction on $m$.

Question. Is there a geometric/homological interpretation of the general Dwork congruences? 


\section{Acknowledgements}

This work was partially supported by the 2008 Starting Grant of College of Science, NTU, Grant 97-2115-M-002-018 of NSC, and a grant of NTU, Taiwan.

\section{Appendix A. Hadamard products}

Here we describe how to obtain the unit roots precisely for certain Hadamard products considered in $[6, \S 3]$. The only missing piece in loc.cit. is to determine the constant $\epsilon_{4}$ in Proposition 2.7 there. To do this, we study the Frobenius action on the cohomology of the totally degenerate fiber by applying the weight spectral sequence in [5]. For references of Hadamard products and examples of pencils of elliptic curves we discuss here, see $[6, \S \S 3.1$ and 3.2].

Let $X, Y \rightarrow \mathbb{P}^{1}$ be two pencils of elliptic curves over a finite field $k$ of characteristic $p$ with totally degenerate fibers $X_{0}, Y_{0}$ at 0 , respectively. We assume that $X_{0}, Y_{0}$ are strictly normal crossing divisors. Let $\xi_{1}$ and $\xi_{2}$ be local bases at 0 of horizontal sections of the relative $H_{\text {cris }}^{1}$ of $X$ and $Y$ over $\mathbb{P}^{1}$, respectively. Then $\xi_{i}$ are eigenvectors of the relative Frobenius. Denote by $c_{i}$ the corresponding eigenvalues. Then $c_{1}=1$ if the degenerate curve $X_{0}$ is of split multiplicative type over $k ; c_{1}=-1$ if $X_{0}$ is non-split. The corresponding statement for $c_{2}$ is similar.

Geometrically, the Hadamard product comes from the following commutative diagram with squares 1 and 2 being Cartesian:

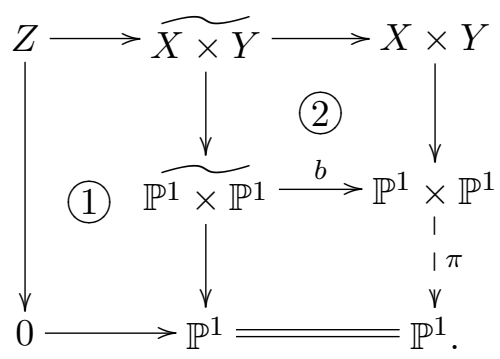

Here $\pi$ is the (coordinate-wise) multiplication; $b$ is the blow-up of $\mathbb{P}^{1} \times \mathbb{P}^{1}$ along $(\infty, 0)$ and $(0, \infty)$.

Over $\bar{k}$, let $Z^{(i)}$ be the disjoint union of all possible intersections of $i$ distinct irreducible components of $Z$. Then we have the weight spectral 
sequence $([5,3.23])$ :

(A.1)

$$
E_{1}^{-j, i+j}=\bigoplus_{r \geq 0, r \geq-j} H_{c r i s}^{i-j-2 r}\left(Z^{(1+j+2 r)} / W\right)(-j-r) \Longrightarrow H^{i}\left(Z^{\times} / W^{\times}\right),
$$

which degenerates at $E_{2}$ modulo torsion ([5, Theorem 3.32]). Here $W$ is the ring of Witt vectors of $k$ and the target $H^{i}\left(Z^{\times} / W^{\times}\right)$is the $i$ th logarithmic crystalline cohomology of $Z$.

Now assume that $X$ and $Y$ are ordinary and have trivial crystalline cohomology groups of odd degrees. Then the weights of the $E_{1}$-terms are all integers and the non-zero terms of $E_{1}$ appear only when the weights are even.

Lemma A.1. With assumptions as above, let $\xi$ be the Hadamard product of $\xi_{i}$ and $c$ the eigenvalue of the relative Frobenius action on $\xi$. Then $c=c_{1} c_{2}$.

Proof. Let $d: E_{1}^{2,0} \rightarrow E_{1}^{3,0}$ be the boundary map in (A.1). Then $c$ represents the relative Frobenius action on the cokernel of $d$. Let $K$ be the field of fractions of $W$. By [5, Lemme 5.2], we see that $(\operatorname{coker} d) \otimes K$ is one-dimensional and the Frobenius acts on $\xi$ as the product of its actions on $\xi_{i}$. Thus the statement follows.

\section{References}

[1] G. Almkvist and W. Zudilin, Differential equations, mirror maps and zeta values, Mirror symmetry $V, 481-515$, AMS/IP Studies in Advance Mathematics, 38, American Mathematical Society, Providence, RI, 2006.

[2] P. Deligne, Local behavior of Hodge structures at infinity, Mirror symmetry II, 683-699, AMS/IP Studies Advance Mathematics, 1, American Mathematical Society, Providence, RI, 1997.

[3] K. Kato, Logarithmic structures of Fontaine-Illusie, Algebraic Analysis, Geometry, and Number Theory (Baltimore, MD, 1988), Johns Hopkins University Press, Baltimore, MD, 1989, 191-224.

[4] N. M. Katz, Travaux de Dwork, Séminaire Bourbaki, 24ème anné (1971/1972), Exp. No. 409, Lecture Notes in Mathematics, 317, Springer, Berlin, 1973, 167-200.

[5] A. Mokrane, La suite spectrale des poids en cohomologie de Hyodo-Kato, Duke Math. J. 72(2) (1993), 301-337. 
[6] K. Samol and D. van Straten, Frobenius polynomials for Calabi-Yau equations, Commun. Number Theory Phys. 2(3) (2008), 537-561.

[7] J. Stienstra and F. Beukers, On the Picard-Fuchs equation and the formal Brauer group of certain elliptic K3-surfaces, Math. Ann. 271(2) (1985), 269-304.

[8] M. van der Put and M. F. Singer, Galois theory of linear differential equations, Grundlehren der Mathematischen Wissenschaften, 328, SpringerVerlag, Berlin, 2003.

[9] J.-D. Yu, Variation of the unit root along the Dwork family of CalabiYau varieties, Math. Ann. 343(1) (2009), 53-78.

Department of Mathematics

National TAIWAN University

TAIPEI 10617

TAIWAN

E-mail address: jdyu@math.ntu.edu.tw

ReCEIVED November 4, 2008 
